\title{
La cesárea electiva en recién nacidos de término se asocia a mayor morbilidad respiratoria neonatal
}

\author{
Elective cesarean in term infants is associated with more neonatal respiratory mortality
}

\section{Objetivo}

Investigar la asociación entre el nacimiento por cesárea electiva, la morbilidad respiratoria neonatal y la edad gestacional al momento del nacimiento.

\section{Diseño y Lugar}

Cohorte prospectiva con datos obtenidos de nacimientos del Servicio de Obstetricia y Neonatología del Hospital Universitario Aarhus en Dinamarca.

\section{Pacientes}

Todos los recién nacidos sin malformaciones, con edad gestacional entre 37 y 41 semanas, nacidos entre 1998 y 2006 .

\section{Medidas principales del resultado}

Morbilidad respiratoria (taquipnea transitoria, síndrome de distrés respiratorio, hipertensión pulmonar persistente del recién nacido) y morbilidad respiratoria severa (necesidad de oxígeno-terapia por más de dos días, de presión positiva continua de la vía aérea o de ventilación mecánica).

\section{Resultados}

2687 neonatos nacidos por cesárea fueron comparados con nacidos por vía vaginal. Esta comparación evidenció un riesgo aumentado de morbilidad respiratoria en aquellos nacidos por cesárea. Estos resultados no se modificaron después del ajuste por la presencia o no de diabetes, tabaquismo o consumo de alcohol materno; ni por la paridad, el índice de masa corporal, el estado civil, la edad o la escolaridad materna; ni por el antecedente de retardo de crecimiento intrauterino y/o presentación pelviana.

Tabla 1: riesgo de morbilidad respiratoria luego de una cesárea electiva de acuerdo a la edad gestacional.

\begin{tabular}{|c|c|c|}
\hline \multirow[t]{2}{*}{ Edad gestacional } & \multicolumn{2}{|c|}{$\begin{array}{c}\text { Morbilidad respiratoria en los nacidos por cesárea } \\
\text { electiva (OR e IC95\%) }\end{array}$} \\
\hline & General & Severa \\
\hline 37 semanas & $3,9(2,4$ a 6,5$)$ & $5,0(1,6$ a 16,0$)$ \\
\hline 38 semanas & $3,0 \quad(2,1$ a 4,3$)$ & $4,2(1,6$ a 11$)$ \\
\hline 39 semanas & $1,9(1,2$ a 3$)$ & $2,4(0,5$ a 12$)$ \\
\hline
\end{tabular}

\section{Conclusiones}

Comparados con neonatos nacidos por vía vaginal, aquellos de término nacidos por cesárea electiva presentan un riesgo mayor de morbilidad general y respiratoria severa, aumentando éste a medida que disminuye la edad gestacional.

Palabras claves: cesárea electiva, morbilidad respiratoria, estudio de cohorte. Key words: elective caesarean section, respiratory morbidity, cohort study. Fuentes de financiamiento: Universidad de Aarhus. No se reportan conflictos de interés.

\section{Comentario}

En los años recientes se ha observado un importante aumento en el número de cesáreas, tanto en nuestro país como a nivel mundial, llegando en muchos centros a representar el $50 \%$ del total de nacimientos. La creencia de que la cesárea podría tener un beneficio para la madre y/o el recién nacido no tiene fundamentos sólidos; y por el contrario, ya hay estudios que muestran un aumento en la morbilidad neonatal asociado a cesáreas electivas al termino del embarazo ${ }^{123}$.

En este estudio se evaluó la asociación entre cesárea electiva al término del embarazo y la presencia de morbilidad respiratoria en el recién nacido, confirmando sus resultados lo observado en otras investigaciones. Un punto a destacar es que la mayor morbilidad neonatal persistió hasta las 39 semanas de edad gestacional, a diferencia de de otros estudios, en los que sólo se había observado hasta las 37 y 38 semanas.

\section{Conclusiones de la comentadora}

La cesárea electiva al término del embarazo es una práctica riesgosa para el neonato ya que se asocia a un incremento del riesgo de problemas respiratorios. A la luz de la tendencia mundial observada de incremento de la tasa de nacimientos por cesárea, los importantes datos de este estudio reafirman la necesidad de implementar acciones para disminuir el número de cesáreas innecesarias, que superan ampliamente la tasa del $15 \%$ recomendada por la Organización Mundial de la Salud.

Una estrategia nacional de atención al parto normal que preserve el protagonismo de la mujer y aumente la calidad de la atención al parto en las instituciones sanitarias podría contribuir en este sentido.

Amorina Pardo [ Servicio de Neonatologia del Departamento de Pediatria Hospital Italiano de Buenos Aires. Amorina.pardo@ hospitalitaliano.org.ar ]

Pardo A. La cesárea electiva en recién nacidos de término se asocia a mayor morbilidad respiratoria neonatal. Evid. actual. práct. ambul. 11(6): 174 Nov-dic 2008. Comentado de: Kirkeby Hansen A y col. Risk of respiratory morbidity in term infants delivered by elective caesarean section: cohort study, BMJ 2008;336:85-87 (12 January), doi:10,1136/bmj,39405,539282,BE. PMID 18077440.pr).

\section{Referencias}

1. Villar J y col. Caesarean delivery rates and pregnancy outcomes: the 2005 WHO global survey on maternal and perinatal health in Latin America.Lancet 2006;367:1819-29.

2. Grisaru S y col. Primary nonmedically indicated cesarean section. Evidence based or modern vogue?.Clin Perinatol 2004, 31:409-30.

3. Pardo A y col. Cesarean delivery at term in low risk pregnancies: effects on neonatal morbidity. PAS Annual Meeting, Hawai, May 2008. 